\title{
The Effects of Prior Stress on Anxiety-Like Responding to Intra-BNST Pituitary Adenylate Cyclase Activating Polypeptide in Male and Female Rats
}

\author{
S Bradley King', Kim R Lezak², Micaela O'Reilly', Donna J Toufexis', William A Falls', Karen Braas ${ }^{3}$, \\ Victor May ${ }^{3}$ and Sayamwong E Hammack*,I \\ 'Department of Psychological Science, University of Vermont, Burlington, VT, USA; ${ }^{2}$ Department of Psychiatry, Harvard University and McLean \\ Hospital, Belmont, MA, USA; ${ }^{3}$ Department of Neurological Sciences, University of Vermont College of Medicine, Burlington, VT, USA
}

\begin{abstract}
Chronic or repeated exposure to stressful stimuli can result in several maladaptive consequences, including increased anxiety-like behaviors and altered peptide expression in anxiety-related brain structures. Among these structures, the bed nucleus of the stria terminalis (BNST) has been implicated in emotional behaviors as well as regulation of hypothalamic-pituitary-adrenal (HPA) axis activity. In male rodents, chronic variate stress (CVS) has been shown to increase BNST pituitary adenylate cyclase activating polypeptide (PACAP) and its cognate $\mathrm{PACl}$ receptor transcript, and BNST PACAP signaling may mediate the maladaptive changes associated with chronic stress. Here, we examined whether CVS would sensitize the behavioral and/or endocrine response to a subthreshold BNST PACAP infusion. Male and cycling female rats were exposed to a 7 day CVS paradigm previously shown to upregulate BNST PACI receptor transcripts; control rats were not stressed. Twenty-four hours following the last stressor, rats were bilaterally infused into the BNST with a normally subthreshold dose of PACAP. We found an increase in startle amplitude and plasma corticosterone levels 30 min following intra-BNST PACAP infusion in male rats that had been previously exposed to CVS. CVS did not enhance the startle response in cycling females. Equimolar infusion of the VPACI/2 receptor ligand vasoactive intestinal polypeptide (VIP) had no effect on plasma corticosterone levels even in previously stressed male rats. These results suggest that repeated exposure to stressors may differentially alter the neural circuits underlying the responses to intra-BNST PACAP, and may result in different anxiety-like responses in males and females.

Neuropsychopharmacology (2017) 42, 1679-1687; doi:10.1038/npp.2017.16; published online I5 February 2017
\end{abstract}

\section{INTRODUCTION}

Multiple physiological and behavioral systems are recruited to promote homeostasis and survival in response to stressor exposure, including the sympathetic arm of the autonomic nervous systems, the hypothalamic-pituitary-adrenal (HPA) axis and central nervous system (CNS) circuits underlying stress-related behavioral changes. Severe or repeated stressor exposure, however, can result in the sustained activation of these response systems, which has been argued to lead to the maladaptive morphological and functional changes that may underlie pathological fear- and anxiety-related states, including altered peptide expression in the brain structures involved in emotional behavior (Hammack et al, 2009; Hammack and May, 2015; Pego et al, 2008; Schulkin et al, 1998; Vyas et al, 2003). Thus, chronic stress may increase vulnerability towards anxiety disorders by altering neural

\footnotetext{
*Correspondence: Professor SE Hammack, Department of Psychological Science, University of Vermont, John Dewey Hall, 2 Colchester Avenue, Burlington, VT 05405-0134, USA, Tel: 802656 I04I, Fax: 802656 8783, E-mail: shammack@uvm.edu

Received 6 September 2016; revised 9 January 2017; accepted 10 January 2017; accepted article preview online 20 January 2017
}

plasticity within stress-responsive CNS nuclei associated with emotional behavior.

Activation of the bed nucleus of the stria terminalis (BNST) has been argued to mediate both stress responding and anxiety-like behavioral responding to temporally distant, long-duration and/or unpredictable threats and may be responsible for mediating anxiety-like emotional states (Hammack et al, 2015; Waddell et al, 2006; Walker et al, 2003). Indeed, stimulation of the BNST mimics many of the physiological responses elicited by anxiogenic stimuli (reviewed in Hammack et al, 2010). Furthermore, anxiogenic pharmacological agents increase BNST neuronal activation (Singewald et al, 2003) and many anxiety-like behaviors are abolished by BNST inactivation (reviewed in Walker et al, 2003). BNST activity has been implicated in anxious temperament among non-human primates (Kalin et al, 2005) and humans (Somerville et al, 2010; Straube et al, 2007), suggesting that altered BNST functioning may be critical for the etiology of several stress-related anxiety disorders (Hammack et al, 2009, 2010; Lebow and Chen, 2016; Pego et al, 2008; Schulkin et al, 1998; Vyas et al, 2003).

In rodents, repeated exposure to stressors increases anxiety-like behavior, and enhances plasticity in the BNST, 
including increased neuronal peptide expression (Hammack et al, 2009; Roman et al, 2014; Stout et al, 2000), enhanced dendritic branching and length, and total BNST volume (Pego et al, 2008; Vyas et al, 2003), and enhanced excitatory synaptic transmission (Daniel and Rainnie, 2016; Dumont et al, 2005, 2008). These findings suggest that chronic stress may promote pathological anxiety-like states, and disorders by enhancing neuronal function and plasticity within the BNST.

Several stress-related peptides have been implicated in regulating BNST activity, and we have implicated BNST pituitary adenylate cyclase activating polypeptide (PACAP) and its cognate G-protein-coupled PAC1 receptor (PAC1R) in mediating the consequences of chronic stress. Neuronal PACAP and PAC1R expression is associated with multiple levels of stressor responding, and the activation of PAC1Rs is both neurotrophic and excitatory in these stress-responsive regions in the CNS and periphery (reviewed in Hammack et al, 2010; Hammack and May, 2015; Smith and Eiden, 2012). Both PACAP and PAC1R transcripts are selectively upregulated in the dorsal aspect of the anterolateral BNST (dBNST), a peptide-rich region that contains the BNST oval nucleus, following chronic variate stress (CVS; Hammack et al, 2009). Moreover, intra-BNST PACAP infusion mimics, whereas intra-BNST PACAP receptor antagonism prevents many of the consequences of chronic stress, suggesting that BNST PACAP activation is both necessary and sufficient for stress- and anxiety-like responding (Hammack et al, 2009; Kocho-Schellenberg et al, 2014; Lezak et al, 2014; Roman et al, 2014).

As noted above, PACAP signaling can have neurotrophic consequences, and we have shown that PAC1 transcripts are upregulated by chronic stress. Here, we examined the effects of prior stress on the anxiety-like behavioral and/or corticosterone response to a subthreshold intra-BNST PACAP infusion in both males and cycling female rats. We demonstrate that stress can potentiate the behavioral and endocrine response to intra-BNST PACAP in males, but not females, suggesting that repeated exposure to stressors may differentially alter the BNST PACAP circuits underlying stress responding in males and females, and may produce sex-dependent differences in anxiety-like responding.

\section{MATERIALS AND METHODS}

\section{Animals}

Adult male $(n=108)$ and adult cycling female $(n=32)$ Sprague-Dawley rats were obtained from Charles River Laboratories (Canada; 200-275 g). Rats were single-housed, maintained on a $12 \mathrm{~h}$ light/dark cycle (lights on at $0700 \mathrm{~h}$ ), and food and water were available ad libitum. Rats were allowed at least 1 week of habituation in their home cages before any experimentation. All procedures were approved by the Institutional Animal Care and Use Committee at the University of Vermont.

\section{Surgical Procedures}

For intra-BNST infusions, rats were anesthetized with isoflurane vapor (1.5-3.5\%), and secured in a stereotaxic apparatus (David Kopf Instruments, Tujunga, CA) with 'blunted' earbars. A midline head incision was made to expose and clean the skull. Four screws were inserted into burr holes to provide skullcap stability. Two stainless steel guide cannulae (26 gauge, PlasticsOne, Roanoke, VA) were lowered at a $20^{\circ}$ angle toward the midline to a point just above the anterolateral BNST, using the following coordinates from Bregma in $\mathrm{mm} ; \mathrm{AP}=-0.1, \mathrm{ML}=+3.9$, and $\mathrm{DV}=-5.3$ from the surface of the dura (Paxinos and Watson, 2007). Once in place, stylettes were inserted and a dental cement skullcap was constructed to secure the cannulae and stylettes. Immediately following surgery, rats were subcutaneously administered Lactated Ringer's Solution to provide hydration, and Carprofen $(5 \mathrm{mg} / \mathrm{kg}$ ) (Pfizer) for analgesia. A second dose of Carprofen was administered $24 \mathrm{~h}$ following surgery. All rats were observed and weighed daily during recovery.

\section{Chronic Variate Stress}

Rats were assigned to control or chronically stressed groups randomly (experiments 2 and 3) or based on baseline startle (experiments 1 and 4). Stressed rats underwent a 7 day CVS paradigm consisting of five different stressors (oscillation, forced swim, footshock, restraint, and pedestal; Hammack et al, 2009). All rats were exposed to a single stressor in the same order at approximately the same time each day (Table 1).

\section{Startle Apparatus}

Each acoustic startle stabilimeter chamber consisted of an $8 \mathrm{~cm} \times 15 \mathrm{~cm} \times 15 \mathrm{~cm}$ acrylic and wire-mesh cage with four stainless steel floor bars spaced $18 \mathrm{~mm}$ apart. The cage was suspended between compression springs within an acrylic frame located within a $90 \mathrm{~cm} \times 70 \mathrm{~cm} \times 70 \mathrm{~cm}$ ventilated sound-attenuating cubical. Chamber movement resulted in the displacement of an accelerometer (Model U321AO2; PCB Piezotronics, Depew, NY), which was fixed to the bottom of the cage; the resulting voltage was proportional to the velocity of displacement. The analog output of the accelerometer was amplified (PCB Piezotronics, Model $483 \mathrm{~B} 21$ ) and digitized on a scale of $0-10 \mathrm{~V}$ by an InstruNET analog to digital converter (GW Instruments, Model 100B; Somerville, MA) interfaced to a Macintosh G3 computer. Startle amplitude was defined as the maximal peak-to-trough

Table I Chronic Variate Stress

\begin{tabular}{lll}
\hline Day & Stressor & Duration \\
\hline 1 & Oscillation & $30 \mathrm{~min}$ \\
2 & Swim & $5 \mathrm{~min}$ \\
3 & Footshock & $5 \mathrm{~s}(\times 2)$ \\
4 & Restraint & $60 \mathrm{~min}$ \\
5 & Pedestal & $30 \mathrm{~min}$ \\
6 & Swim & $5 \mathrm{~min}$ \\
7 & Footshock & $5 \mathrm{~s}(\times 2)$ \\
\hline
\end{tabular}

Stressed rats were exposed to a 7 day CVS paradigm in which one of the five different stressors was presented each day. Two stressors (swim and footshock) were repeated. 
voltage during the first $200 \mathrm{~ms}$ after the stimulus onset. Startle responses were evoked by $50 \mathrm{~ms}$ white-noise bursts generated by a Macintosh G3 computer sound file, amplified by a Radio Shack Amplifier (100 W; Model MPA-200; Tandy, Fort Worth, TX), and delivered through a high frequency speaker (Radio Shack Super-Tweeter; Tandy, Fort Worth, TX) located $5 \mathrm{~cm}$ from the back of each cage.

\section{Corticosterone Enzyme-Linked Immunoassay}

Following collection of plasma from tail nick blood samples, a corticosterone enzyme-linked immunoassay (CORT EIA, Enzo Life Sciences, Farmingdale, NY) was used to determine plasma corticosterone levels. Plasma samples were first diluted $1: 30$ and heated in a $75^{\circ} \mathrm{C}$ water bath for $\sim 1 \mathrm{~h}$ to denature corticosterone binding globulin (Lezak et al, 2014). The sensitivity of the assay was $27 \mathrm{pg} / \mathrm{ml}$.

\section{Vaginal Cytology and Tracking of Estrous Phase}

Vaginal cytology was examined daily until it was verified that each subject cycled through all phases of the estrous cycle. Rats were lightly restrained and loose vaginal cells were removed with cotton tipped applicators soaked in sterile water and rolled onto slides. Swabs were taken daily at the same time that animals were weighed and handled (0900 hour). On infusion days, swabs were collected after all experimentation was completed to mitigate any confounds related to the stress of collecting the cells. The slides were examined with a light microscope and the estrous phase was assessed. Each phase of the estrous cycle was identified using the following characteristics: proestrus is marked by epithelial cell nuclei, estrus is marked by clumped cornified cells, and diestrus is marked by leukocytes with scattered epithelial cells (Bangasser and Shors, 2008). All females included cycled normally, even those exposed to stressors.

\section{Perfusion and Histology}

Upon completion of each experiment, rats were anesthetized with sodium pentobarbital $(\sim 195-245 \mathrm{mg} / \mathrm{kg})$ and perfused transcardially with $0.9 \%$ saline containing $0.1 \%$ heparin (Sagent, Schaumburg, IL) followed by $10 \%$ formalin. Brains were then removed and postfixed for $24 \mathrm{~h}$ in $10 \%$ formalin. Fixed tissue was sectioned at $60 \mu \mathrm{m}$ on a cryostat and mounted on subbed slides for staining with cresyl violet. Following staining, slides were coverslipped and cannulae placements were verified using a $\times 4$ Nikon Objective on an Olympus light microscope. There was no significant notable tissue damage following infusion, consistent with our prior reports (Hammack et al, 2009; Kocho-Schellenberg et al, 2014; Lezak et al, 2014; Roman et al, 2014).

\section{Statistics}

Statistical analyses were completed using SPSS version 21 (IBM Software, Armonk, NY) and all graphical representations were completed using GraphPad Prism version 6 (GraphPad Software, San Diego, CA). Rats were eliminated from analysis if cannulae placement fell outside the histological boundary of the BNST or if their startle amplitude or corticosterone level was more than two standard deviations away from the treatment mean. Twoway ANOVA (all experiments) or Repeated Measures ANOVA (experiment 1 and 4) was used to calculate overall group differences and interactions followed by Tukey's posthoc comparisons. A trending interaction in experiment 2 was further probed with subsequent $t$-test.

\section{Experimental Procedure}

Experiment 1-effects of intra-BNST PACAP infusion following CVS on anxiety-like behavior in males. We have previously shown that our CVS paradigm does not alter baseline startle activity; however, CVS does enhance acoustic startle responding to treatments that already elevate startle in a BNST-dependent manner, including bright lights and handling (Hammack et al, 2009). Additionally, we've shown that CVS selectively increases PACAP/PAC1 transcripts in the dBNST (Hammack et al, 2009). These findings suggest that the CVS-induced sensitization in BNST-dependent startle requires some initial BNST stimulation to be observed, and could depend on a sensitization in the BNST PACAP system.

Following postoperative recovery, all rats were administered two baseline startle test on consecutive days in which they were placed into the startle chambers and allowed a 5 min acclimation to the chamber. The response to 33 randomized noise burst (described above) that varied in intensity $(95,100$, or $105 \mathrm{~dB} ; 11$ of each stimulus) with a $30 \mathrm{~s}$ intertrial interval was then determined as the average response across the session. On the basis of the average startle across all three noise burst for the 2 days of baseline startle rats were 'matched' into CVS $(n=18)$ or control groups $(n=18)$ so that baseline startle amplitude did not differ between groups. For the subsequent 7 days, stressed rats were treated with CVS as described above; control rats were handled and weighed but not otherwise stressed. On the 8th day, before infusions, all rats were returned to the startle chambers and received a third startle test that was identical to the prior baseline testing sessions. Immediately after this third test, rats were removed from the startle chamber and bilaterally infused with either $0.5 \mu \mathrm{l} 0.05 \%$ bovine serum albumin (BSA) vehicle (Santa Cruz Biotechnology, Santa Cruz, CA) or $0.5 \mu \mathrm{g}$ PACAP38 (American Peptide, Sunnyvale, CA) in $0.5 \mu \mathrm{l}$ BSA vehicle. Mean startle amplitude across test 3 was used to assign animals to PACAP or vehicle groups. Groups were assigned to minimize differences in startle levels before infusion (Supplementary Figure 1). The PACAP dose was chosen as we have previously shown it to be below the threshold required to increase acoustic startle responding or increase corticosterone release when infused into the BNST (Hammack et al, 2009; Lezak et al, 2014). Infusions were completed in a separate room under light restraint. For each infusion: the stylette was removed and an internal cannula that extended $1 \mathrm{~mm}$ beyond the end of the guide cannula was inserted. Injections were completed using a $10 \mu \mathrm{l}$ Hamilton syringe connected to the internal cannula with PE50 plastic tubing. A volume of $0.5 \mu \mathrm{l}$ was infused into the BNST over the course of $60 \mathrm{~s}$. Following infusion, the internal cannula was left in place for an additional $60 \mathrm{~s}$ to allow for diffusion of the drug away from the infusion site. After infusions rats were returned to the home cage for 15 min until subsequent testing. 
Fifteen minutes after infusion, the rats were returned to the startle chamber and after a $5 \mathrm{~min}$ acclimation period, rats were administered a fourth startle test that consisted of 72 startle stimuli that varied in intensity. After this fourth test, rats were returned to the colony until where they remained until perfusions and histology were performed (described above).

Experiment 2-effects of intra-BNST PACAP infusion following CVS on circulating corticosterone. Following postoperative recovery, rats were weighed and pseudorandomly assigned to control $(n=21)$ or CVS $(n=18)$ groups. Control rats were handled and weighed daily, and housed in their home cages until infusion; stressed rats underwent the 7 days of CVS described above. Twenty-four hours after the last stressor, animals were weighed and bilaterally infused into the BNST with vehicle or PACAP38 as described above. Following bilateral infusion, the rat was returned to the home cage and remained in the colony room for $30 \mathrm{~min}$ until blood sampling.

Blood sampling was conducted in a nearby room at $30 \mathrm{~min}$ post infusion via tail nick. The time required to transport animals and obtain the blood sample was $<3 \mathrm{~min}$, to avoid contamination of the sample with the increase in corticosterone release associated with cage removal. This time point was chosen because we have previously shown increased corticosterone following intra-BNST PACAP $(1 \mu \mathrm{g})$ at 30-60 min post infusion (Lezak et al, 2014). The sample was immediately placed into a refrigerated centrifuge and spun between 2000 and 4000 r.p.m. for 15-20 min to separate the plasma. Plasma was then collected and stored at $-20^{\circ} \mathrm{C}$ until corticosterone quantification via a CORT EIA (see above). Following blood collection, the animals were returned to the colony where they remained until perfusions and histology were performed (described above).

Experiment 3-effects of intra-BNST VIP infusion following CVS on circulating corticosterone. Although only PACAP binds with high affinity to the PAC1 receptor, PACAP and VIP bind with relatively equal affinities to VPAC1 and VPAC2 receptors (Ishihara et al, 1992; see Vaudry et al, 2009 for review). We have previously found VIP to have no effect on the PACAP response (Missig et al, 2014; Roman et al, 2014). In experiment 3, we assessed whether CVS could potentiate the response to BNST VIP infusion or if VIP could produce an endocrine response on its own (Missig et al, 2014; Roman et al, 2014). All methods and procedures were identical to experiment 2 . vehicle groups (control/vehicle, $n=8$; CVS/vehicle, $n=8$ ) received $0.5 \mu \mathrm{l} 0.05 \% \mathrm{BSA} /$ vehicle. VIP groups (control/VIP, $n=8$; CVS/VIP, $n=9$ ) received

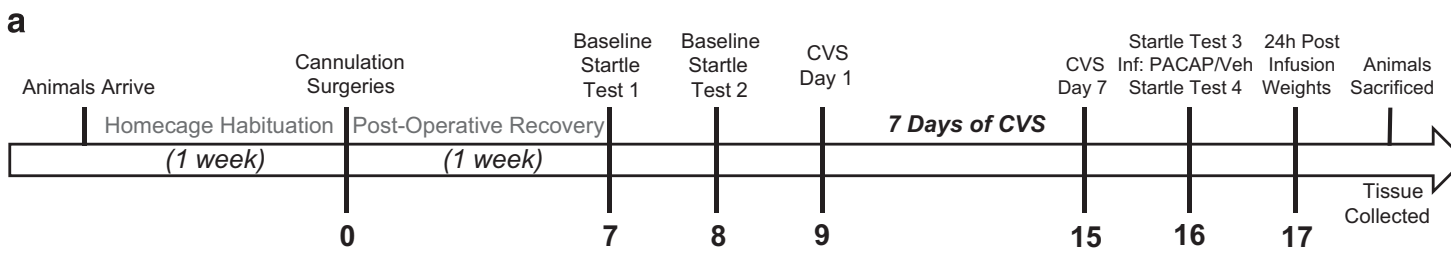

b

\begin{tabular}{|c|c|c|c|c|c|}
\hline Animals Arrive & $\begin{array}{l}\text { nulation } \\
\text { geries }\end{array}$ & $\begin{array}{l}\text { CVS } \\
\text { Day } 7\end{array}$ & $\begin{array}{l}\text { Inf: PACAP/Veh } \\
\text { 30m Post-Inf: } \\
\text { Tailnick \& blood } \\
\text { collection }\end{array}$ & $\begin{array}{l}24 \mathrm{~h} \text { Post } \\
\text { Infusion } \\
\text { Weights }\end{array}$ & $\begin{array}{r}\text { Animals } \\
\text { Sacrificed }\end{array}$ \\
\hline Homecage Habituation & Post-Operative Recovery & 7 Days of CVS & & & \\
\hline (1 week) & (1 week) & & & & \\
\hline & 0 & 13 & 14 & 15 & $\begin{array}{c}\text { Tissue } \\
\text { Collected }\end{array}$ \\
\hline
\end{tabular}

C
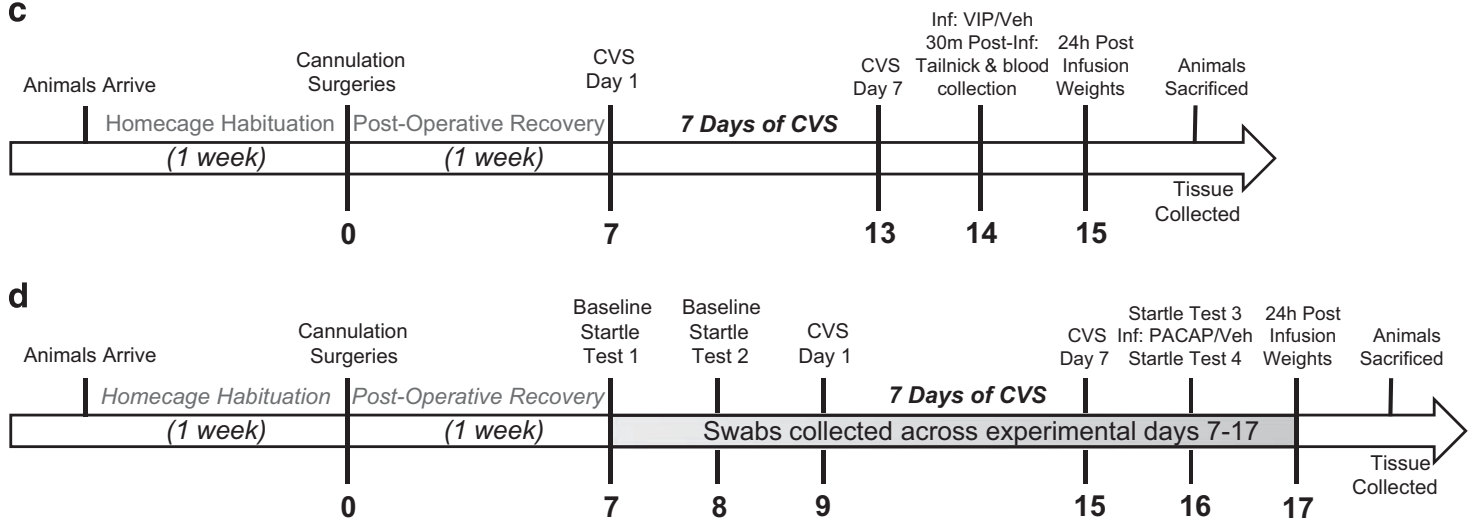

Figure I Timeline of experimental procedure. Rats were allowed I week of home cage habituation before bilaterally BNST cannulation surgeries. Following I week of postoperative recovery, rats were administered two baseline startle tests. Twenty-four hours after the second startle test, rats underwent 7 days of CVS. Twenty-four hours after the last stressor rats were administered a third startle test and then immediately infused with PACAP. Fifteen minutes after infusion, rats were administered a final startle test (a). In experiments 2 and 3, rats were matched into control or stress groups after postoperative recovery, and exposed to the same 7 days of CVS. Twenty-four hours after the last stressor rats were bilaterally infused with PACAP (b) or VIP (c) and tail blood was collected 30 min after infusion for a corticosterone ELIZA. Experiment 4 followed an identical procedure to experiment I, but vaginal swabs were collected across experimental days $7-17$ (d). 

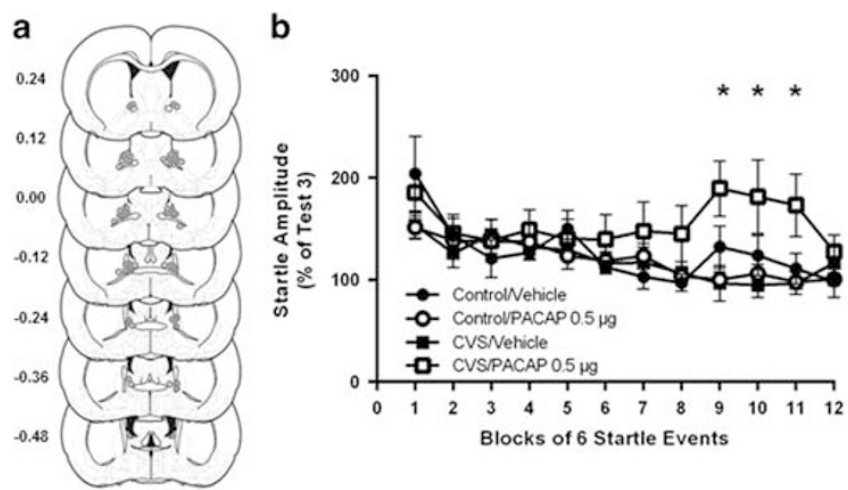

Figure 2 (a) Estimates of center of PACAP infusion for experiment I. Open dots represent site(s) of drug infusion depicted on coronal sections as a distance $(\mathrm{mm})$ from Bregma (histological figures modified from Paxinos and Watson (2007)). (b) BNST PACAP infusion increased startle amplitude but only in rats previously exposed to CVS. Data represent test 4 startle amplitude (post infusion) as a percent of test 3 (pre-infusion) startle. Significant three-way Blocks $\times$ CVS $\times$ PACAP interaction, $F \quad(I 3$, $403)=2.084, p=0.01$. The effects of PACAP vary across test blocks. Data represents mean $+1-$ SEM. *Significantly different at $p<0.05$.

$0.73 \mu \mathrm{g}$ VIP in $1 \mu \mathrm{l}$ of vehicle; a dose that was equimolar $(221 \mu \mathrm{M})$ to the PACAP38 dose used in all other experiments. Plasma was collected and stored at $-20^{\circ} \mathrm{C}$ until corticosterone quantification via a CORT EIA.

Experiment 4-effects of intra-BNST PACAP infusion following CVS on anxiety-like behavior in cycling females. As mentioned above, little is known about how stress alters the BNST PACAP system in cycling females. Thus, experiment four was conducted to determine how chronic stress affects the anxiety-like behavioral response to intraBNST PACAP in cycling females. All methods and procedures were identical to experiment 1 . In addition, vaginal swabs were taken across the 11 days of the experiment, starting on day 1 of baseline startle, and ending $24 \mathrm{~h}$ after the fourth startle test (Figure 1d) to evaluate the role of estrous and estrogen (E2) in these responses (described above). Mean startle across the two days of baseline testing was used to 'match' animals into CVS $(n=14)$ or control $(n=11)$ groups. Twenty-four hours after the last stressor a third startle test was conducted before the animals were placed into treatment groups, infused and administered the fourth startle test as described above. As in experiment 1, groups were assigned to minimize differences in startle amplitudes before infusion (Supplementary Figure 2). After this fourth test, rats were returned to the colony where they remained until perfusions and histology were performed (described above).

\section{RESULTS}

\section{Experiment 1-CVS Potentiates the Anxiety-Like Behavioral Response to Intra-BNST PACAP Infusion in Males}

We have previously shown CVS to upregulate PACAP transcript and peptide levels, in addition to PAC1 transcript levels, specifically within the BNST (Hammack et al, 2009), which may mediate the maladaptive consequences associated with chronic stress. This stress-induced upregulation suggest that the BNST may be more sensitive to subsequent PACAP release following CVS, resulting in exaggerated anxiety-like responses. To assess this, we bilaterally cannulated adult male rats for intra-BNST PACAP38 infusions $(0.5 \mu \mathrm{g} / 0.5 \mu \mathrm{l})$ and then measured the anxiety-like startle response.

Startle amplitude was reassessed following startle test 3 and before infusions. In agreement with our previous findings (Hammack et al, 2009), we did not find an effect of CVS on baseline startle (test 3 startle amplitude; $M_{\text {control }}=124.95$, $\left.M_{\mathrm{CVS}}=120.87\right), \quad t \quad(32)=0.22, \quad p=0.82 \quad$ (Supplementary Figure 3 ).

The centers of BNST infusion sites for PACAP treated rats are depicted in Figure 2a. We have previously shown this dose to be subthreshold, producing no effect on its own, however, as can be seen in Figure $2 b$, this dose significantly increased startle amplitude, but only in rats that received prior stress. The data are presented as startle amplitude across the first 12 blocks of startle test 4 (post-PACAP infusion) as a percent of startle test 3 (pre-PACAP infusion). Repeated measures analysis of variance across the first 12 three-minute test blocks revealed a significant three-way Blocks $\times$ CVS $\times$ PACAP interaction across all test bins, $\mathrm{F}(13,403)=2.084, p=0.01$. Hence, CVS elevated baseline startle amplitude to a subthreshold intra-BNST PACAP infusion, which is consistent with our prior report suggesting that CVS enhances the acoustic startle response to bright lights (Hammack et al, 2009). Post-hoc comparisons showed significant difference in groups at blocks 9, 10, and 11 ( $\sim 30 \mathrm{~m}$ post infusion). There were no statistically significant main effects of CVS or PACAP, supporting our prior findings that CVS does not enhance acoustic startle responding on its own, and the effects of CVS on acoustic startle responding can only be observed following BNST stimulation.

\section{Experiment 2-CVS Potentiates the Corticosterone Response to Intra-BNST PACAP Infusion in Males}

Experiment 1 showed that a subthreshold dose of PACAP38 following chronic stress increased anxiety-like behavior. To further characterize the effects of prior stress on the response to intra-BNST PACAP, we used procedures identical to experiment 1, but measured the corticosterone response. Cannula placements for infusions are shown in Figure 3a.

We found an increase in plasma corticosterone $30 \mathrm{~min}$ after intra-BNST PACAP infusion, but like experiment 1, this was only observed in the rats previously exposed to CVS (Figure 3b). Two-way ANOVA revealed a significant main effect of CVS, F $(1,37)=13.05, p<0.001$, and a significant main effect of PACAP, F $(1,37)=7.016, p=0.01$, on plasma corticosterone levels. In addition, results revealed a trending interaction between CVS and PACAP on plasma corticosterone levels, $\mathrm{F}(1,37)=3.401, p=0.07$. Further examination of the trending interaction with subsequent $t$-test revealed significant differences in plasma corticosterone levels between the CVS/PACAP and CVS/vehicle groups, $t(17)=3.53, p<0.01$ as well as the CVS/PACAP and no stress/PACAP groups, $t(17)=3.53, p<0.01$. There was no significant difference in corticosterone levels between the no stress/PACAP and no stress/vehicle groups, $t(20)=0.54$, ns. The results of the $t$-tests support the interaction between CVS and PACAP, suggesting that, like the behavioral 


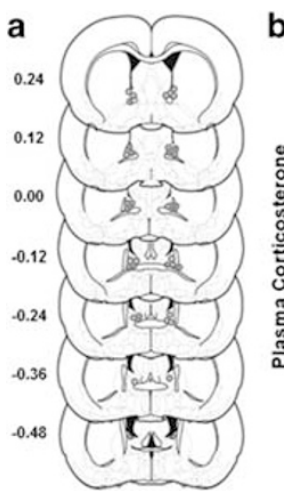

\section{b}

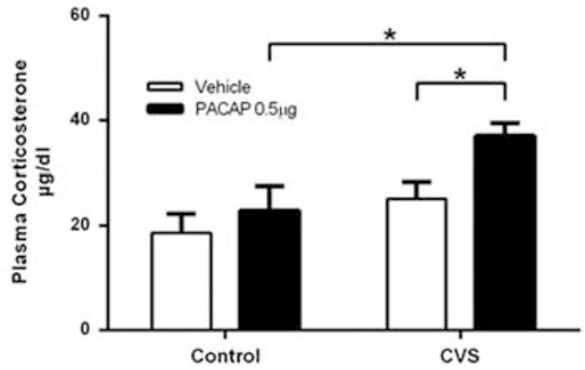

Figure 3 (a) Estimates of center of PACAP infusion for experiment 2. Open dots represent site(s) of drug infusion depicted on coronal sections as a distance $(\mathrm{mm})$ from Bregma (histological figures modified from Paxinos and Watson (2007)). (b) Plasma corticosterone was elevated $30 \mathrm{~m}$ after BNST PACAP infusion, but only in rats exposed to CVS, suggesting that stress also potentiates the endocrine response to intra-BNST PACAP infusion. Main effect of CVS, F $(I, 37)=13.05, p<0.05$ and PACAP, $F(I, 37)=7.061, p<0.05$. Data represents mean $+/-$ SEM. * Significantly different at $p<0.05$.

response in experiment 1 , CVS also sensitizes the corticosterone response to intra-BNST PACAP infusion.

\section{Experiment 3-Intra-BNST VIP Infusion Following CVS has no Effect on Circulating Corticosterone}

PACAP effects can be mediated by three receptor subtypes. PAC1Rs are selective for PACAP, whereas VPAC1/2Rs show equally high affinity for PACAP and VIP. In experiment 3, we assessed whether stress could potentiate the corticosterone response to intra-BNST VIP, or if VIP produced a response on its own.

Using procedures identical to experiment 2, adult male rats were infused with VIP following CVS and plasma corticosterone was measured $30 \mathrm{~min}$ after infusion. Power analysis revealed similar levels of power to that of experiment 2 . Infusion sites for VIP-treated animals are depicted in Figure 4a. Two-way analysis of variance revealed no effect of CVS, F $(1,29)=0.04$, ns, and no effect of VIP, F (1, $29)=0.001$, ns, on plasma corticosterone levels (Figure $4 \mathrm{~b}$ ). Furthermore, there was no significant interaction between CVS and VIP on corticosterone levels, F $(1,29)=2.218$, ns, suggesting that the potentiated responses observed in experiments 1 and 2 were likely mediated via PAC1 receptors.

\section{Experiment 4-CVS Attenuates the Anxiety-Like Behavioral Response to Intra-BNST PACAP Infusion in Females}

The previous experiments suggest that prior stress sensitizes both the behavioral and endocrine response to intra-BNST PACAP in males; as noted above, several lines of evidence suggest that PACAP may interact with E2 to promote anxiety-like behavior (reviewed in Hammack and May, 2015). Hence, we assessed the effects of prior stress on anxiety-like behavior in intact, cycling females while tracking estrous phase across the experiment, using a procedure identical to experiment 1 .

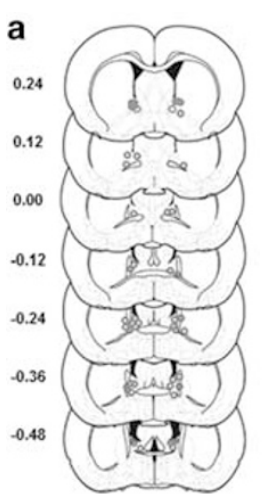

b

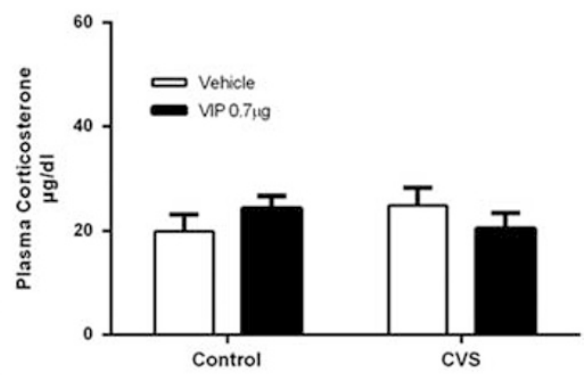

Figure 4 (a) Estimates of center of VIP infusion for experiment 3. Open dots represent site(s) of drug infusion depicted on coronal sections as a distance $(\mathrm{mm})$ from Bregma (histological figures modified from Paxinos and Watson (2007)). (b) Intra-BNST VIP infusion had no effect on plasma corticosterone levels, suggesting that the potentiated endocrine response was mediated via PACIR's. Data represents mean +/ - SEM.

Similar to the results from male rats in experiment 1 , we did not find an effect of CVS on baseline startle in cycling intact female rats (test 3 startle amplitude; $M_{\text {control }}=157.86$, $\left.M_{\mathrm{CVS}}=166.45\right), \quad t \quad(24)=0.25, \quad p=0.80 \quad$ (Supplementary Figure 3).

Cannula placements for females infused with PACAP depicted in Figure 5a. The data are presented as startle amplitude across the first 12 blocks of test 4 after infusion as a percent of startle test 3 (pre-PACAP infusion). Repeated measures analysis of variance across the first 12 three-minute test blocks revealed a significant three-way Blocks $\times$ CVS $\times$ PACAP interaction across all test bins, F $(33,231)=1.478$, $p=0.05$ (Figure 5b). Post-hoc comparisons revealed significant differences at all blocks except 6,10 , and 11 . Two-way ANOVA revealed a significant CVS $\times$ PACAP interaction, $F$ $(1,16)=4.972, p=0.04$, and a significant main effect of CVS, $\mathrm{F}(1,16)=5.980, p=0.03$. Thus, unlike males, PACAP infusion increased startle amplitude but only in control females. Females that were previously stressed showed an attenuated response. These data suggest that in cycling females, the regulation of PACAP responding by prior stress is likely different than that observed in males.

\section{DISCUSSION}

Prior exposure to CVS sensitized both the behavioral and endocrine response to a normally subthreshold infusion of intra-BNST PACAP in male, but not female, rats. Hence, only stressed males showed a reliable increase in startle amplitude and plasma corticosterone levels $30 \mathrm{~min}$ after a normally subthreshold dose of intra-BNST PACAP. CVS did not enhance the startle response to a subthreshold dose of PACAP in normally cycling, intact females. The time course of the anxiogenic effects of BNST PACAP was consistent with our prior reports (Hammack et al, 2009; Lezak et al, 2014) as well as the time course of intra-BNST CRHenhanced startle (Lee and Davis, 1997). We further demonstrated that the enhanced responses were likely driven by BNST PAC1R activation as equimolar infusion of the VPAC1/2R agonist, VIP, had no effect on plasma 


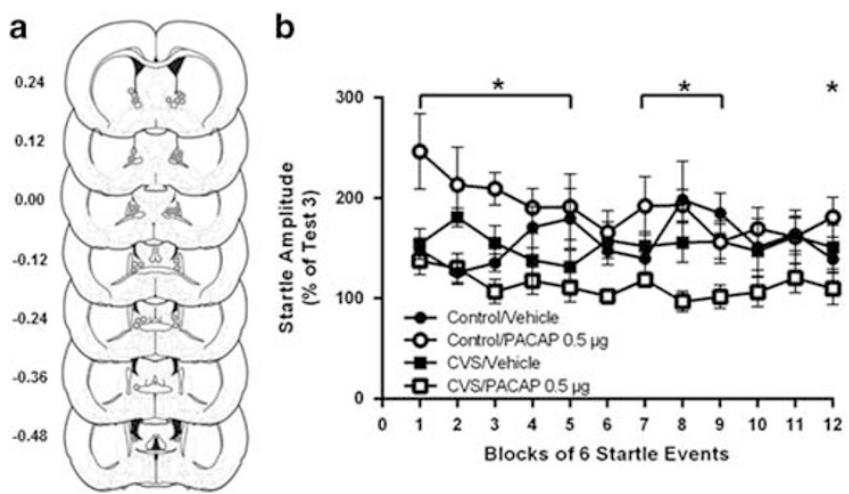

Figure 5 (a) Estimates of center of PACAP infusion for experiment 4. Open dots represent site(s) of drug infusion depicted on coronal sections as a distance $(\mathrm{mm})$ from Bregma (histological figures modified from Paxinos and Watson (2007)). (b) BNST PACAP infusion increased startle amplitude but only in control females. CVS females showed an attenuated response. Data represent test 4 startle amplitude (post infusion) as a percent of test 3 (pre-infusion) startle. Significant Blocks $\times$ CVS $\times$ PACAP, and CVS $\times$ PACAP interactions, $F(33,23 I)=1.48, F(I, 16)=4.97, p<0.05$. Significant main effect of CVS, $F(I, 16)=5.98, p<0.05$. Data represents mean $+/-$ SEM. *Significantly different at $p<0.05$.

corticosterone even in stressed rats. These results suggest that stress-induced plasticity within the BNST via sensitized PACAP/PAC1 signaling may have a critical role in mediating the maladaptive consequences of chronic stress. Furthermore, the current results suggest that stress may mediate this system differently in males and females.

Similar sexual divergences (ie, sex differences that emerge following an environmental change or event-stressor/threat exposure) have been observed in the CRH system following stress. For example, Bangasser and colleagues have shown sex difference in $\mathrm{CRH}_{1}$ receptor function; hence, following stress females have decreased coupling of $\beta$-arrestin to $\mathrm{CRH}_{1}$ receptors and enhanced coupling to $\mathrm{Gs}$, rendering females more responsive to acute stress and less able to adapt to chronic stress (Bangasser et al, 2010). Additional research suggest analogous sex difference may occur with other seven transmembrane G-protein-coupled receptor systems (reviewed in Valentino et al, 2013). Furthermore, sexbiased signaling may result in divergent behaviors. For example, under normal, unstressed conditions males and females show similar performance on a standard sustained attention task (SAT); however, following central administration of CRH, task performance decreases in both sexes, but females are more impaired than males, and this was dependent on estrous stage (Cole et al, 2016). Hence, stress may result in sexual divergences in multiple neuropeptide/ transmitter systems which are further influenced by the estrous cycle. These sex difference may underlie the increased prevalence of stress-related disorders in females.

Roman et al (2014) showed that PACAP antagonism blocked the sensitized portion of the corticosterone response to the open field maze (OFM). That is, PACAP antagonism blunted the enhanced corticosterone response observed in chronically stressed animals, suggesting that stress may have sensitized the BNST PACAP system. The current results corroborate and extend these findings, as male rats that had been stressed showed both a sensitized behavioral and corticosterone response to a subthreshold intra-BNST PACAP infusion.
As noted above, there have been several reports suggesting that chronic exposure to stressors, stress hormones and/or pharmacological treatments enhances multiple indices of neural plasticity within the BNST (Daniel and Rainnie, 2016; Dumont et al, 2005; Hammack et al, 2009, 2010; Herman, 2013; Makino et al, 1994; Pego et al, 2008; Roman et al, 2014; Schulkin et al, 1998; Vyas et al, 2003). The microcircuitry in the BNST is complex, and the activation of different BNST subregions can be associated with both increased and decreased stress and emotional responding (Gungor and Paré, 2016; Jennings et al, 2013; Kim et al, 2013). Moreover, while BNST neurons primarily use $\gamma$-aminobutyric acid (GABA; Daniel and Rainnie, 2016; Nguyen et al, 2016), a distinct and functionally relevant population of BNST neurons appears to be glutamatergic (Jennings et al, 2013; Nguyen et al, 2016), and a number of neuropeptides and hormones are often coexpressed with GABA/glutamate in BNST neurons (Dabrowska et al, 2013; Daniel and Rainnie, 2016; Nguyen et al, 2016). It is more likely that stressor exposure, potentially via PACAP, enhances synaptic function in specific BNST pathways and neuronal populations to promote stress- and anxiety-like responding, rather than ubiquitously throughout the nucleus. The pathways affected remain to be determined; however current techniques that allow for the targeting of specific neuronal populations will aid in this investigation.

PACAP binds to three G-protein-coupled PACAP receptor subtypes with PAC1 showing the highest affinity for PACAP; moreover, PAC1Rs show signaling properties related to both repair and neurotrophic signaling. Uniquely, multiple isoforms of the PAC1R results from alternative splicing. The various $\mathrm{PAC} 1 \mathrm{R}$ subtypes are expressed in specific regions throughout the CNS and can be differentially coupled to G $\alpha$ s (engaging adenylyl cyclase) and/or G $\alpha \mathrm{q}$ (engaging phospholipase C) to activate signaling pathways which enhance calcium mobilization, membrane depolarization, action potential frequency, and neurotransmitter synthesis and release (reviewed in Vaudry et al, 2009). These mechanisms together with arrestin-driven endosome signaling following PAC1R internalization can also engage mitogen-activated protein kinase kinase/extracellular signal-regulated kinase and phosphatidylinositol 3-kinase/Akt pathways, which appear critical for trophic signaling during neurodevelopment, survival, repair, and regeneration following injury and neuroplasticity following physiological challenges (Ohtaki et al, 2006; Reglodi et al, 2012). Furthermore, PACAP/ PAC1R signaling can enhance the expression and function of other growth factor mechanisms, including brain-derived neurotrophic factor (BDNF) and tropomyosin receptor kinase B (TrkB; Braas et al, 2007; Yaka et al, 2003) and has been shown to mediate the neurotrophic (ie, neurite length and number) actions of BDNF (Ogata et al, 2015). Moreover, CNS BDNF expression is diminished in PAC1 receptor knockout animals (Zink et al, 2004), and we have reported that PACAP treatment upregulates BDNF and TrkB transcripts in BNST explants (Hammack et al, 2010). Chronic stress results in long-term structural and functional changes in dBNST neuronal plasticity and BDNF function has been associated with anxiety-related behaviors, implicating PACAP-stimulated BDNF expression as one of the mechanisms underlying stress-induced plasticity within this structure. 
PACAP, like CRH, is widely distributed throughout the HPA axis and sympathetic nervous system, and functions as one of the principal mediators of the both the endocrine and behavioral response to stressful events. Furthermore, PACAP-positive terminals have been shown to innervate and form synapses with $\mathrm{CRH}$-positive neurons in many stress-related brain regions, including both the PVN and BNST. In the PVN, PACAP can stimulate $\mathrm{CRH}$ production and secretion, and studies from PACAP null mice have shown that PVN CRH mRNA is not upregulated following restraint stress (Stroth and Eiden, 2010). Thus, PACAP signaling is upstream of CRH function in the PVN and also likely upstream of CRH in the BNST (Kozicz et al, 1997). Hence, repeated stress may increase PACAP signaling and/or PAC1Rs on populations of $\mathrm{CRH}$ neurons in the BNST to increase stress- and anxiety-like responding.

Women are more than twice as likely as their male counterparts to develop PTSD, and in addition to psychosocial explanations, differences in the physiological systems that underlie the emotional behavior and stress responses may also have a role. Levels of PACAP and a SNP within an ERE for the PAC1 receptor gene is correlated with PTSD in women but not men, suggesting that PACAP dysregulation may have a role in the higher prevalence of PTSD in women (Ressler et al, 2011). Moreover, we have shown that E2 treatment elevates BNST PACAP and PAC1 transcripts in rats (Ressler et al, 2011), and BNST PACAP infusion interacts with $\mathrm{E}$ to reduce food intake (Kocho-Schellenberg et al, 2014). The current results suggest that anxiety-like behavior may depend on interactions between stress, estrous/ E2 and PACAP. Post-hoc correlation analyses revealed that estrous phase on the last day of stress was likely related to test 3 startle (before infusion) and test 4 startle (post-infusion startle), but due to a lack of statistical power, neither of these was significant, $p>0.05$. It seems likely that changes in acoustic startle responding across the cycle could contribute to variability across experimental groups. Interestingly, subsequent moderation analyses revealed that post infusion anxiety level (startle) was a function of both PACAP and estrous: females that were in proestrus on the last day of stress that also exhibited enhanced startle before infusion showed attenuated anxiety-like behavior following PACAP infusion, whereas those in proestrus but did not exhibit elevated startle before infusion showed an enhanced startle response following BNST PACAP that was similar to males (interaction 1: pre-infusion startle $\times$ cycle, $p<0.001$; interaction 2: pre-infusion startle $\times$ PACAP, $p<0.001$; interaction $3 /$ both: $p=0.001)$. Females in other phases of the cycle showed basal level startle responses following BNST PACAP infusion regardless of prior stress. These analyses suggest that the level of stress and phase of estrus before PACAP infusion differentially mediates the PACAP effects in females.

In aggregate, the current results support the hypothesis that repeated stressor exposure physically alters the neurochemistry, morphology, and physiology associated with increases in fearand anxiety-like behavior to alter function and plasticity within the BNST. Moreover, stress may differentially mediate the BNST PACAP system in males and females, suggesting that the BNST PACAP system may represent the plasticity underlying stress-related disorders and may be one mechanism driving the discrepancies between prevalence rates in human psychiatric diseases, implicating the BNST PACAP system as a potential therapeutic target in these stress-related psychopathologies. Although suggestive, how E2 and estrus might interact with PACAP to influence anxiety-like behavior and produce pathological states is still unclear, awaiting future investigations.

\section{FUNDING AND DISCLOSURE}

This work was supported by Grant MH-97988 (SEH) from the National Institutes of Health. The authors declare no conflict of interest.

\section{ACKNOWLEDGMENTS}

We thank Katy Alpogianis, Douglas Vormstein-Schneider, and Beniah Brumbaugh for their assistance in the laboratory.

\section{REFERENCES}

Bangasser DA, Curtis A, Reyes BA, Bethea TT, Parastatidis I, Ischiropoulos $\mathrm{H}$ et al (2010). Sex differences in corticotropinreleasing factor signaling and trafficking. Mol Psychiatry 15: 877-877.

Bangasser DA, Shors TJ (2008). The bed nucleus of the stria terminalis modulates learning after stress in masculinized but not cycling females. J Neurosci 28: 6383-6387.

Braas KM, Schutz KC, Bond JP, Vizzard MA, Girard BM, May V (2007). Microarray analyses of pituitary adenylate cyclase activating polypeptide (PACAP)-regulated gene targets in sympathetic neurons. Peptides 28: 1856-1870.

Cole RD, Kawasumi Y, Parikh V (2016). Corticotropin releasing factor impairs sustained attention in male and female rats. Behav Brain Res 296: 30-34.

Dabrowska J, Hazra R, Guo J-DD, Li C, Dewitt S, Xu J et al (2013). Striatal-enriched protein tyrosine phosphatase-STEPs toward understanding chronic stress-induced activation of corticotrophin releasing factor neurons in the rat bed nucleus of the stria terminalis. Biol Psychiatry 74: 817-826.

Daniel SE, Rainnie DG (2016). Stress modulation of opposing circuits in the bed nucleus of the stria terminalis. Neuropsychopharmacology 41: 103-125.

Dumont EC, Mark GP, Mader S, Williams JT (2005). Selfadministration enhances excitatory synaptic transmission in the bed nucleus of the stria terminalis. Nat Neurosci 8: 413-414.

Dumont ÉC, Rycroft BK, Maiz J, Williams JT (2008). Morphine produces circuit-specific neuroplasticity in the bed nucleus of the stria terminalis. Neuroscience 153: 232-239.

Gungor NZ, Paré D (2016). Functional heterogeneity in the bed nucleus of the stria terminalis. J Neurosci 36: 8038-8049.

Hammack SE, May V (2015). Pituitary adenylate cyclase activating polypeptide in stress-related disorders: data convergence from animal and human studies. Biol Psychiatry 78: 167-177.

Hammack SE, Todd TP, Kocho-Schellenberg M, Bouton ME (2015). Role of the bed nucleus of the stria terminalis in the acquisition of contextual fear at long or short context-shock intervals. Behav Neurosci 129: 673-678.

Hammack SE, Cheung J, Rhodes KM, Schutz KC, Falls WA, Braas $\mathrm{KM}$ et al (2009). Chronic stress increases pituitary adenylate cyclase-activating peptide (PACAP) and brain-derived neurotrophic factor (BDNF) mRNA expression in the bed nucleus of the stria terminalis (BNST): roles for PACAP in anxiety-like behavior. Psychoneuroendocrinology 34: 833-843.

Hammack SE, Roman CW, Lezak KR, Kocho-Shellenberg M, Grimmig B, Falls WA et al (2010). Roles for pituitary adenylate cyclase-activating peptide (PACAP) expression and signaling in the bed nucleus of the stria terminalis (BNST) in mediating the 
behavioral consequences of chronic stress. J Mol Neurosci 42: 327-340.

Herman JP (2013). Neural control of chronic stress adaptation. Front Behav Neurosci 7: 61.

Ishihara T, Shigemoto R, Mori K, Takahashi K, Nagata S (1992). Functional expression and tissue distribution of a novel receptor for vasoactive intestinal polypeptide. Neuron 8: 811-819.

Jennings J, Sparta D, Stamatakis A, Ung R, Pleil K, Kash T et al (2013). Distinct extended amygdala circuits for divergent motivational states. Nature 496: 224-228.

Kalin NH, Shelton SE, Fox AS, Oakes TR (2005). Brain regions associated with the expression and contextual regulation of anxiety in primates. Biol Psychiatry 58: 796-804.

Kim S-YY, Adhikari A, Lee SY, Marshel JH, Kim CK, Mallory CS et al (2013). Diverging neural pathways assemble a behavioural state from separable features in anxiety. Nature 496: 219-223.

Kocho-Schellenberg M, Lezak KR, Harris OM, Roelke E, Gick N, Choi I et al (2014). PACAP in the BNST produces anorexia and weight loss in male and female rats. Neuropsychopharmacology 39: 1614-1623.

Kozicz T, Vigh S, Arimura A (1997). Axon terminals containing PACAP-and VIP-immunoreactivity form synapses with CRFimmunoreactive neurons in the dorsolateral division of the bed nucleus of the stria terminalis in the rat. Brain Res 767: 109-119.

Lebow MA, Chen A (2016). Overshadowed by the amygdala: the bed nucleus of the stria terminalis emerges as key to psychiatric disorders. Mol Psychiatry 21: 450-463.

Lee Y, Davis M (1997). Role of the hippocampus, the bed nucleus of the stria terminalis, and the amygdala in the excitatory effect of corticotropin-releasing hormone on the acoustic startle reflex. J Neurosci 17: 6434-6446.

Lezak KR, Roelke E, Harris OM, Choi I (2014). Pituitary adenylate cyclase-activating polypeptide (PACAP) in the bed nucleus of the stria terminalis (BNST) increases corticosterone in male and female rats. Psychoneuroendocrinology 45: 11-20.

Makino S, Gold P, Schulkin J (1994). Effects of corticosterone on $\mathrm{CRH}$ mRNA and content in the bed nucleus of the stria terminalis; comparison with the effects in the central nucleus of the amygdala and the paraventricular nucleus of the hypothalamus. Brain Res 657: 141-149.

Missig G, Roman CW, Vizzard MA, Braas KM, Hammack SE, May V (2014). Parabrachial nucleus (PBn) pituitary adenylate cyclase activating polypeptide (PACAP) signaling in the amygdala: implication for the sensory and behavioral effects of pain. Neuropharmacology 86: 38-48.

Nguyen AQ, Dela Cruz JA, Sun Y, Holmes TC, Xu X (2016). Genetic cell targeting uncovers specific neuronal types and distinct subregions in the bed nucleus of the stria terminalis. J Comp Neurol 524: 2379-2399.

Ogata K, Shintani N, Hayata-Takano A, Kamo T, Higashi S, Seiriki K et al (2015). PACAP enhances axon outgrowth in cultured hippocampal neurons to a comparable extent as BDNF. PLoS ONE 10: e0120526.

Ohtaki H, Nakamachi T, Dohi K (2006). Pituitary adenylate cyclaseactivating polypeptide (PACAP) decreases ischemic neuronal cell death in association with IL-6. Proc Natl Acad Sci USA 103: 7488-7493.

Paxinos G, Watson C. The Rat Brain in Stereotaxic Coordinates. 6th edn. Elsevier: New York, 2007.
Pego JM, Morgado P, Pinto LG (2008). Dissociation of the morphological correlates of stress-induced anxiety and fear. Eur J Sci 27: 1503-1516.

Reglodi D, Kiss P, Szabadfi K, Atlasz T, Gabriel R, Horvath G et al (2012). PACAP is an endogenous protective factor-insights from PACAP-deficient mice. J Mol Neurosci 48: 482-492.

Ressler KJ, Mercer KB, Bradley B, Jovanovic T, Mahan A, Kerley K et al (2011). Post-traumatic stress disorder is associated with PACAP and the PAC1 receptor. Nature 470: 492-497.

Roman CW, Lezak KR, Hartsock MJ, Falls WA, Braas KM, Howard AB et al (2014). PAC1 receptor antagonism in the bed nucleus of the stria terminalis (BNST) attenuates the endocrine and behavioral consequences of chronic stress. Psychoneuroendocrinology 47: 151-165.

Schulkin J, Gold PW, McEwen BS (1998). Induction of corticotropin-releasing hormone gene expression by glucocorticoids: implication for understanding the states of fear and anxiety and allostatic load. Psychoneuroendocrinology 23: 219-243.

Singewald N, Salchner P, Sharp T (2003). Induction of c-Fos expression in specific areas of the fear circuitry in rat forebrain by anxiogenic drugs. Biol Psychiatry 53: 275-283.

Smith CB, Eiden LE (2012). Is PACAP the major neurotransmitter for stress transduction at the adrenomedullary synapse? J Mol Neurosci 48: 403-412.

Somerville LH, Whalen PJ, Kelley WM (2010). Human bed nucleus of the stria terminalis indexes hypervigilant threat monitoring. Biol Psychiatry 68: 416-424.

Stout SC, Mortas P, Owens MJ, Nemeroff CB (2000). Increased corticotropin-releasing factor concentrations in the bed nucleus of the stria terminalis of anhedonic rats. Eur J Pharmacol 401: 39-46.

Straube T, Mentzel HJ, Miltner W (2007). Waiting for spiders: brain activation during anticipatory anxiety in spider phobics. Neuroimage 37: 1427-1436.

Stroth N, Eiden LE (2010). Stress hormone synthesis in mouse hypothalamus and adrenal gland triggered by restraint is dependent on pituitary adenylate cyclase-activating polypeptide signaling. Neuroscience 165: 1025-1030.

Valentino R, Bangasser D, Bockstaele E (2013). Sex-biased stress signaling: the corticotropin-releasing factor receptor as a model. Mol Pharmacol 83: 737-745.

Vaudry D, Falluel-Morel A, Bourgault S, Basille M (2009). Pituitary adenylate cyclase-activating polypeptide and its receptors: 20 years after the discovery. Pharmacol Rev 61: 283-357.

Vyas A, Bernal S, Chattarji S (2003). Effects of chronic stress on dendritic arborization in the central and extended amygdala. Brain Res 965: 290-294.

Waddell J, Morris RW, Bouton ME (2006). Effects of bed nucleus of the stria terminalis lesions on conditioned anxiety: aversive conditioning with long-duration conditional stimuli and reinstatement of extinguished fear. Behav Neurosci 120: 324-336.

Walker DL, Toufexis DJ, Davis M (2003). Role of the bed nucleus of the stria terminalis versus the amygdala in fear, stress, and anxiety. Eur J Pharmacol 463: 199-216.

Yaka R, He DY, Phamluong K, Ron D (2003). Pituitary adenylate cyclase-activating polypeptide (PACAP (1-38)) enhances $\mathrm{N}$ methyl-D-aspartate receptor function and brain-derived neurotrophic factor expression. J Biol Chem 278: 9630-9638.

Zink M, Otto C, Zörner B, Zacher C, Schütz G, Henn FA et al (2004). Reduced expression of brain-derived neurotrophic factor in mice deficient for pituitary adenylate cyclase activating polypeptide type-I-receptor. Neurosci Lett 360: 106-108.

Supplementary Information accompanies the paper on the Neuropsychopharmacology website (http://www.nature.com/npp) 